\title{
Essential Role of the Hippocampal Formation in Rapid Learning of Higher-Order Sequential Associations
}

\author{
Ceren Ergorul and Howard Eichenbaum \\ Center for Memory and Brain, Boston University, Boston, Massachusetts 02215
}

Evidence from studies of amnesia and functional imaging in humans suggest that the medial temporal lobe is involved in the learning of higher-order sequences during acquisition of serial reaction time (SRT) tasks. However, these studies have not clarified whether the hippocampal formation specifically is essential to this type of learning. Here, we developed a rodent model of the SRT task to examine the specific roles of the hippocampal formation in learning first- and second-order conditional sequences. Selective hippocampal formation lesions speeded performance and impaired accuracy on both first- and second-order sequences. Additionally, whereas controls distinguished the sequences based on their complexity alone, animals with hippocampal lesions initially differentiated sequences only by their length. Over multiple sessions, hippocampal rats gradually differentiated sequences by their complexity and not length, similar to control subjects. These findings indicate that the hippocampal formation itself plays an essential role in rapid acquisition of higher-order sequence representations. Extrahippocampal systems can also acquire complex sequential representations, albeit via a gradual learning mechanism.

Key words: serial reaction time; SRT; FOC; SOC; MTL; multiple memory systems; NMDA lesions; excitotoxic lesions; sequence learning; rat

\section{Introduction}

Several theoretical perspectives have suggested a key role for the hippocampus in memory for sequential events (Tulving, 1972; Levy, 1989; Wallenstein et al., 1998; Lisman, 1999) and substantial experimental evidence supports this notion (Agster et al., 2002; Burgess et al., 2002; Fortin et al., 2002; Kesner et al., 2002; Lee and Wilson, 2002; Ergorul and Eichenbaum, 2004). One paradigm that has been used extensively to study sequence learning is the serial reaction time (SRT) test, a task in which subjects decrease their reaction times as they repeat finger-tapping sequences (Knopman and Nissen, 1987). Some early studies reported that patients with Alzheimer's disease and amnesia consequent to damage in the medial temporal lobe (MTL) were not impaired in SRT and instead suggested involvement of cortical and basal ganglia systems (Knopman and Nissen, 1991; Jackson et al., 1995). Other evidence indicated that the MTL plays an important role in explicit expression of knowledge about the sequence-order in SRT (Reber and Squire, 1994, 1998; DeCoteau and Kesner, 2000; Hopkins et al., 2004).

Curran (1997) found that amnesic patients were impaired even in implicit SRT tasks when subjects must learn higher-order predictive sequences. This study compared SRT tasks that in-

Received Dec. 8, 2005; revised March 8, 2006; accepted March 11, 2006.

This work was supported by National Institute of Mental Health Grant MH52090, National Institute on Aging Grant AG09973, and National Science Foundation Grant SBE-0354378. We thank Joseph R. Manns for writing the Q-Basic codes for the present study, as well as for comments on the protocols and results. We thank Joseph D. Lippi, Andrea L. Harris, and Margaret R. Oliner for their assistance in animal testing, and Sean J. Wright for analyzing the sequences for the study. We also thank Michael A. Christie for his comments and Norbert J. Fortin for statistical assistance.

Correspondence should be addressed to Prof. Howard Eichenbaum, Center for Memory and Brain, Boston University, 2 Cummington Street, Boston, MA 02215. E-mail: hbe@bu.edu.

DOI:10.1523/JNEUROSCI.0441-06.2006

Copyright $\odot 2006$ Society for Neuroscience $\quad$ 0270-6474/06/264111-07\$15.00/0 volved first-order conditional (FOC) sequences, in which the occurrence of each item was unambiguously predicted by the immediately preceding item, versus second-order conditional (SOC) sequences, in which successive items could be predicted only from multiple preceding items. Controls and amnesic patients showed comparable reaction time reductions for FOC sequences but the amnesic patients were impaired on SOC sequences. Consistent with these findings, Schendan et al. (2003) found activation in the MTL at the earliest stage of both implicit and explicit learning of SOC sequences.

These observations suggest that the MTL may be involved in learning higher-order associations that contribute to sequence memories, and that the difference between first-order and second-order sequences may be critical in understanding the role of the hippocampus in sequence learning. However, the studies on amnesia and those using functional imaging have not clarified precisely which structures of the MTL are critical. The amnesic patients of previous SRT studies had damage to several structures within and outside the MTL (Reber and Squire, 1994, 1998; Curran, 1997; Hopkins et al., 2004). Similarly, the study by Schendan et al. (2003) revealed activation during learning of SOC sequences in several areas within and outside the MTL. Because of recent evidence that implicit forms of associative memory may be supported by temporal cortical areas outside the hippocampal formation (Manns and Squire, 2001) and the suggestion that higher-order sequence learning is mediated by a large network of cortical areas (Keele et al., 2003), it is important to determine whether the hippocampal formation itself is essential to higherorder SRT learning. Here, we developed a rodent model of the SRT task and found that the hippocampal formation itself is critical for the rapid learning of second-order sequences, whereas extrahippocampal areas can also support this learning, albeit at a very gradual rate. 


\section{Materials and Methods}

Subjects

Twenty-eight male Long-Evans rats were used as subjects. All rats were 3-months-old and weighed between 350 and $450 \mathrm{~g}$ before surgery. During the study, rats had ad libitum access to food and had restricted access to water for $30 \mathrm{~min}$ per day during pretraining and testing. Rats were kept on a $12 \mathrm{~h}$ illumination cycle and tested at the light phase.

\section{Lesions}

Rats were anesthetized with halothane (1\%) and nitrous oxide/oxygen (7:3) throughout the surgery. After the rat's head was shaved, it was placed in a stereotaxic instrument (Kopf, Tujunga, CA). We injected $0.081 \mathrm{mg}$ of atropine sulfate (Phoenix Pharmaceutical, St. Joseph, MO) intramuscularly to prevent respiratory complications. The skin covering the skull was incised along the midline, and then the skull was exposed and leveled.

In the surgery of the hippocampal lesion $(\mathrm{H})$ group, initially, a section of the skull overlying the hippocampus was bilaterally removed. Then the dura was poked with a needle and a 30-gauge stainless-steel needle was successively lowered at seven different locations per hemisphere. Before each lesion, the needle was allowed to settle for $10 \mathrm{~s}$, then $100-125 \mathrm{nl}$ of NMDA ( $10 \mathrm{mg} / \mathrm{ml}$ stock solution in PBS, pH 7.2) was injected at a rate of $200 \mathrm{nl} / \mathrm{min}$ with a $5 \mu \mathrm{l}$ syringe (Hamilton, Reno, NV) attached to a microsyringe pump controller (World Precision Instruments, Sarasota, $\mathrm{FL})$. The coordinates and injection volumes were as follows: anteroposterior (AP) $-2.2 \mathrm{~mm}$ (from bregma), mediolateral (ML) $\pm 1.0 \mathrm{~mm}$ (from bregma), dorsoventral (DV) $-3.2 \mathrm{~mm}$ (from dura), $100 \mathrm{nl}$; AP $-3.0 \mathrm{~mm}, \mathrm{ML} \pm 1.8 \mathrm{~mm}, \mathrm{DV}-3.0 \mathrm{~mm}, 100 \mathrm{nl}$; AP $-4.0 \mathrm{~mm}, \mathrm{ML} \pm 2.8$ $\mathrm{mm}, \mathrm{DV}-2.8 \mathrm{~mm}, 100 \mathrm{nl}$; AP $-4.8 \mathrm{~mm}, \mathrm{ML} \pm 4.8 \mathrm{~mm}, \mathrm{DV}-6.7 \mathrm{~mm}$, $125 \mathrm{nl}$; AP $-4.8 \mathrm{~mm}, \mathrm{ML} \pm 4.5 \mathrm{~mm}, \mathrm{DV}-3.5 \mathrm{~mm}, 100 \mathrm{nl}$; AP $-5.7 \mathrm{~mm}$, $\mathrm{ML} \pm 4.9 \mathrm{~mm}, \mathrm{DV}-4.0 \mathrm{~mm}, 100 \mathrm{nl}$; AP $-5.7 \mathrm{~mm}, \mathrm{ML} \pm 5.1 \mathrm{~mm}, \mathrm{DV}$ $-6.0 \mathrm{~mm}, 125 \mathrm{nl}$. The needle was left at each location for $1 \mathrm{~min}$ after the injections. Then, the needle was raised $0.2 \mathrm{~mm}$ above the target and left at this level for $2 \mathrm{~min}$ before it was completely retracted. The surgery protocol for the sham-operated controls was identical to that of the hippocampal lesion group except that the needle was not lowered in the brain and no injection was made.

In all surgeries, rats received a subcutaneous injection of $5 \mathrm{ml}$ of lactated Ringer's solution (Phoenix Pharmaceutical) to prevent dehydration and an intramuscular injection of $0.5 \mathrm{mg}$ of diazepam (Abbott Laboratories, Abbott Park, IL) to prevent postsurgical seizures. At the end of each surgery, the wound was sutured and covered with topical antiseptic (betadine solution; Purdue Frederick, Stamford, CT) followed by an application of a topical antibiotic (Panolog Cream; Fort Dodge Laboratories, Fort Dodge, IA). For $7 \mathrm{~d}$ after surgery, acetaminophen (suspension liquid; CVS, Woonsocket, RI) was mixed with the drinking water to reduce pain and cephalexin oral suspension (40 $\mathrm{mg} / \mathrm{kg}$ per day) was absorbed into the food to prevent any bacterial infection. Rats were allowed to recover for 3 weeks before the pretraining began.

\section{Apparatus}

The behavioral testing apparatus was an aluminum behavioral chamber (50.8 $\mathrm{cm}$ long, $35.6 \mathrm{~cm}$ wide, and $30.5 \mathrm{~cm}$ high) with a concave wall (61 $\mathrm{cm}$ long) on one side. Eight cylindrical nosepoke ports $(1.9 \mathrm{~cm}$ in diameter, $1.9 \mathrm{~cm}$ deep) were equally spaced ( $7.6 \mathrm{~cm}$ from center to center), 5.1 $\mathrm{cm}$ above the floor. A water spout was located at the bottom of each port such that the rat's mouth was in position to receive a water reward after an appropriate nosepoke into the port. In addition, a light-emitting diode (LED) was placed $0.5 \mathrm{~cm}$ above each port. The lid of the chamber was made of aluminum and had an exhaust fan that ran continuously during testing. The floor was $0.635 \mathrm{~cm}$ stainless-steel mesh.

\section{Pretraining}

Water-restricted rats were pretrained through three stages to make nosepokes to illuminated ports to receive water rewards. During pretraining and testing, only the middle six ports were used.

Stage 1. On the first session, a drop of water was placed into each port and all six ports in the apparatus were cued by illumination of the LEDs above. A nosepoke to any cued port was rewarded with a $0.1 \mathrm{~s}$ pulse of water within that port and constituted a trial. A rewarded trial resulted in termination of all cues until the rat removed its nose from the port, then all ports except ports already rewarded on that run were recued and the rat could select any remaining cued ports, and so on, until all cued ports were nosepoked. Nosepokes to any of the uncued ports resulted in termination of all cues until the nosepoke ended and then all ports not yet visited on that trial were recued. After the completion of successful nosepokes to each of the six ports, all six were recued simultaneously to begin the next trial for a total of 10 runs.

Stage 2. The protocol was similar to stage 1 , except that a $5 \mathrm{~s}$ time out, during which all cues were terminated, was implemented after an error. If the rat made a nosepoke during the last $2 \mathrm{~s}$ of the time out, the recuing was delayed for another $5 \mathrm{~s}$. Stage 2 involved 10 runs of six trials each.

Stage 3. Each run consisted of six trials in which the six ports were cued one at a time in pseudorandom order, until all six ports were rewarded. A correct response was defined as a nosepoke to a port within $10 \mathrm{~s}$ after it was cued. An error was defined as a nosepoke to an uncued port and an omission was defined as no poke to any port for $>10 \mathrm{~s}$. Both errors and omissions were followed by a $5 \mathrm{~s}$ time out. After an error or an omission, the same port was recued. After a correct response, the next port was illuminated $1 \mathrm{~s}$ after the rat's nosepoke ended. The reaction time for a nosepoke was measured as the time passed between cuing and a correct nosepoke to that port. The number of runs in a session was gradually increased to 48 over successive days as the rats responded more quickly to the cued ports. Rats were trained over several days for 48 runs per session (288 rewarded responses) until their total number of errors and omissions were $<10 \%(<29)$ in a session. When each rat satisfied this criterion, it was assigned to one of three testing groups (see below).

\section{Testing on fixed sequences}

We examined performance in learning a six-item-long SOC sequence composed from three distinct elements and that of two FOC sequences that were equated with the SOC sequence either for the sequence length [a six-item FOC sequence composed of six elements (FOC6)] or for the number of elements [a three-item FOC sequence composed of three elements (FOC3)]. The protocol was identical to stage 3 of pretraining except that cuing followed a fixed repetitive sequence. All rats were tested on the SOC, FOC6, and FOC3 sequences. To partially counterbalance for the order of testing on the three types of sequence, after pretraining, each rat was assigned to one of three groups, which received training on the sequences in different orders: SOC-FOC3-FOC6, FOC3-SOC-FOC6, or FOC6-FOC3-SOC. Testing on each type of sequence lasted for $12 \mathrm{~d}$. Rats were initially presented with the sequence for five consecutive daily sessions, each of which involved 48 runs of six trials until rats made 288 correct responses. On the sixth day, learning of the sequence was probed by comparing performance on the fixed sequence versus random order sequences. In the first half of the probe session, rats were presented with the sequence until they completed 144 correct trials. Subsequently, in the second half, the same ports were presented in pseudorandom order for another 144 correct trials. From day 7 to day 11, rats were again tested on the same fixed sequence for 288 trials per session, followed by a second probe session on day 12 that was identical to the first probe session. Once testing on a particular sequence was completed, testing for the next type of sequence began on the following day. Testing on the second and third types of sequence included an additional session on the fixed sequence before the first probe session to insure that rats extinguished the previously learned fixed sequence(s) and fully acquired the new one. Data for this additional session were not included in analysis because of the absence of a comparable data set from the first type of sequence tested. Thus, the total duration of testing on sequences considered for analysis was $36 \mathrm{~d}$. Rats were tested every day.

\section{Fixed sequences}

Six-item second-order conditional sequence. This sequence was six items long and involved repetitive cuing of six items (composed from three ports) in the following order: $4-5-6-4-6-5 .$. , where numbers correspond to left-to-right positions in the eight-port array. Every item in the sequence occurred with identical frequency and predicted the other two items on $50 \%$ of the trials each.

Three-item first-order conditional sequence. This sequence provided a 
comparison for the SOC sequence using the same three ports but presented in an FOC where each item predicted the next reliably. The sequence was $5-4-6-5-4-6$. . Note that on each run, the three-item sequence was repeated so that the total number of items in a run was equal to that for SOC and FOC6 sequences.

Six-item first-order conditional sequence. This sequence provided a comparison to the SOC sequence using an FOC sequence length composed of the same number of items but involving additional ports. The sequence was $4-2-3-5-7-6$. .

\section{Random sequences}

Six sets of pseudorandom sequences were used in the second half of the probe sessions. Each set was composed of 144 stimuli and was balanced for the right- and left-side turns as well as for location frequency. Two of the sets, R6A and R6B, involved pseudorandom presentations of six ports and were used on probes with the FOC6 that used the same six ports. The other four sets, R3A-R3D, involved pseudorandom presentations of three ports and were used on probes for SOC and FOC3 sequences and used the same three ports.

\section{Histology}

After postsurgery testing, animals were injected with an overdose of sodium pentobarbital and perfused with saline, followed by $10 \%$ formalin solution. Brains were removed and saturated with $20 \%$ sucrose solution overnight. Fifty-micrometer-thick coronal sections (every fourth) were taken with a cryostat. Next, sections were processed with Nissl staining protocol.

\section{Data analysis}

Data for each fixed sequence were combined from three testing groups that differed in the order of sequence presentations. Data on errors and on the average reaction times were analyzed between groups and among the different types of sequences. For every session, the correct average reaction time (CART) was calculated as the sum of the reaction times for all correct trials divided by the number of correct trials (288). On probe sessions, cARTs were calculated separately for the first and second halves (144 correct trials each).

Additionally, session cART scores were normalized for differences among different sequence types in the distances animals had to traverse between ports. If the distance between centers of any two adjacent ports is represented by the unit $d$, then, in the SOC sequence, the distance covered by the rat for the six transitions in a run was $8 d$. In the FOC3 sequence, the run distance for six transitions was $8 d$, and in FOC6, the run distance for six transitions was $10 \mathrm{~d}$. The average distance covered by the rat for any six transitions on R6A and R6B runs was 9.3 $d$, whereas the average distance for six transitions on R3A and R3C was $8 d$, and on R3B and $\mathrm{R} 3 \mathrm{D}$ the average transition distance was $7.9 d$. Therefore, cART scores were normalized to $8 d$ for every testing session on the fixed sequences as well as on probe sessions.

The data were analyzed with statistical software (StatView; SAS Institute, Cary, NC). Comparisons of group performance were initially performed using repeated measures ANOVAs. Next, paired and unpaired $t$ tests were used for additional comparisons.

\section{Results \\ Histology}

Data for four of these rats were excluded from the study for the following reasons: in one brain, the dorsal hippocampus was unilaterally spared; in another, there was extensive unilateral damage to the anterior portion of the brain outside the hippocampus. Finally, two other brains had extensive damage to the cortex surrounding the hippocampal formation.

Data of the remaining 10 rats with lesions limited to the hippocampal formation (hippocampus proper, the dentate gyrus, subiculum, and entorhinal cortex) were used for the present analyses. These rats had almost complete bilateral lesions of the hippocampus proper and dentate gyrus (Fig. 1). Some damage to the subiculum and to the cortex overlying the dorsal hippocampus
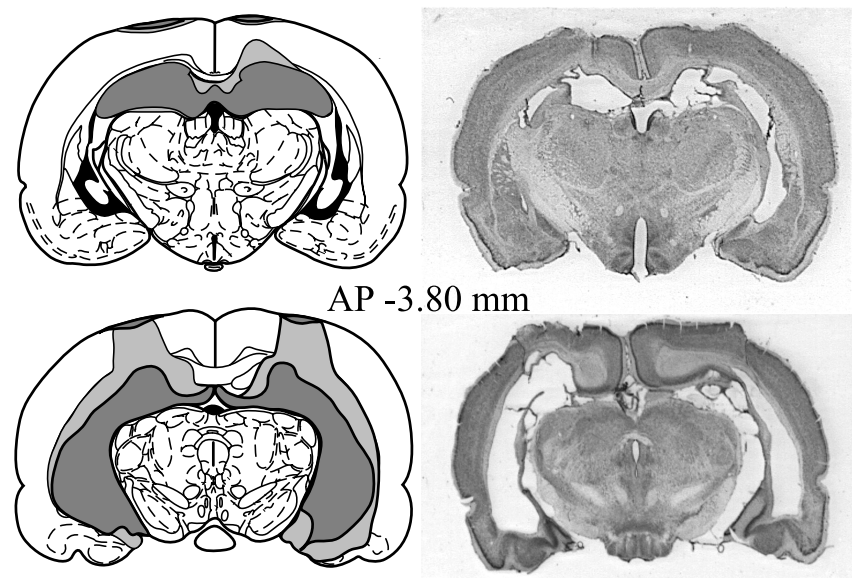

AP $-3.80 \mathrm{~mm}$

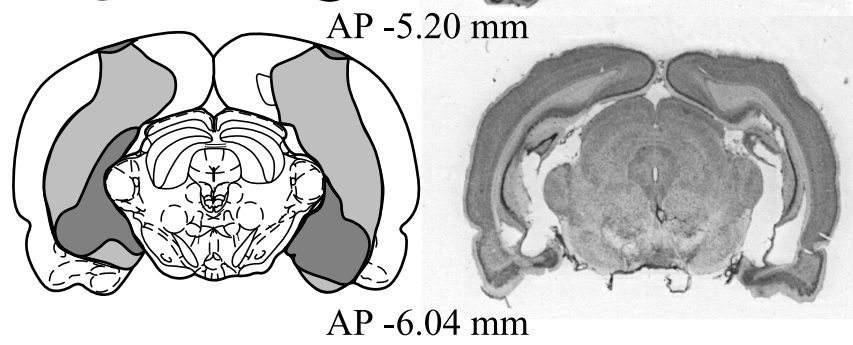

Figure 1. Illustrations of the smallest (dark gray) and largest (light gray) brain lesions for the $\mathrm{H}$ group, and photomicrographs of sections from a representative brain with a hippocampal lesion. Lesions are reconstructed on coronal sections adapted from Paxinos and Watson (1997) at AP $-3.80 \mathrm{~mm},-5.20 \mathrm{~mm}$, and $-6.04 \mathrm{~mm}$.

was observed in all $\mathrm{H}$ subjects. In most of the $\mathrm{H}$ brains, there was damage to the entorhinal cortex, the presubiculum and parasubiculum as well, but not to perirhinal or postrhinal cortical areas. Additionally, three of the rats had unilateral damage to the cortical amygdaloid nucleus. There was also partial unilateral damage to the basolateral amygdala in one of these rats. Postpiriform transition area was damaged in one rat. Finally, there was unilateral damage to the anterodorsal and laterodorsal thalamic nuclei in another rat.

The control group consisted of 14 rats and during surgery no toxin was injected into their brains. Thirteen of the controls had normal brain anatomy with minimal damage to the cortex overlying the dorsal hippocampus. Interestingly, one of the control brains was abnormal such that it had enlarged lateral ventricles surrounded by a thinned cortex. This rat's data were excluded from the analysis.

\section{Pretraining}

All rats initially completed 60 correct responses in both stage 1 and stage 2 of pretraining. At stage 3, when all six ports were illuminated one at a time in pseudorandom order, the controls completed $1926.9 \pm 144.8$ (mean \pm SEM) correct trials until their total number of errors and omissions were $<10 \%(<29)$ in a 288 -trial session. The $\mathrm{H}$ group satisfied the same criterion in $1944 \pm 243.0$ trials. An unpaired $t$ test between groups indicated that groups did not differ in the number of trials to criterion $\left(t_{(21)}=0.06 ; p=0.95\right)$. In the session when the criterion was satisfied, the total number of errors and omissions of controls and the $\mathrm{H}$ group was $22.8 \pm 1.1$ and $23.3 \pm 1.1$, respectively. An unpaired $t$ test confirmed that groups were not different on this measure $\left(t_{(21)}=0.32 ; p=0.75\right)$. Furthermore, there was no difference between groups when the errors and omissions were compared separately in this last session (unpaired $t$ tests: all $p$ 
values $>0.56)$. These results indicate that controls and the $\mathrm{H}$ group acquired stimulus-response (light port) associations at comparable rates. The groups were equally accurate and motivated in responding to random presentations of stimuli. However, in the last pretraining session when the criterion was reached, $\mathrm{H}$ rats' reaction times were $902 \pm 58 \mathrm{~ms}$ (mean \pm SEM), whereas controls' reaction times were $1211 \pm 69 \mathrm{~ms}$ on their correct response trials. An unpaired $t$ test confirmed that $\mathrm{H}$ rats had shorter reaction times than controls in their correct response trials (unpaired $t$ test, $t_{(21)}=3.29$; $p=0.004$ ), indicating that lesions of the hippocampal formation increased response speed.

\section{Testing}

Comparisons between groups

Changes in the cART and in the number of errors were analyzed for 10 sessions on each sequence. These sessions were from the first half (days 1-5) and the second half of training (days 7-11). Data for probe sessions (days 6 and 12) were analyzed separately for each sequence.

Reaction times and errors in learning fixed sequences. A repeated measures ANOVA on cARTs of the $\mathrm{H}$ group and controls did not indicate a significant group by sequence type by session interaction $\left(F_{(18,378)}=1.21 ; p=0.25\right)$. However, a repeated measures ANOVA on cARTs indicated a significant group effect $\left(F_{(1,21)}=16.03 ; p=0.0006\right)$, a significant sequence type effect $\left(F_{(2,42)}=20.03 ; p<0.0001\right)$, and a significant session effect $\left(F_{(9,189)}=60.54 ; p<0.0001\right)$ (Fig. $\left.2 A-C\right)$. Also, a repeated measures ANOVA indicated a trend for a significant interaction between groups versus sequence types in cARTs (group by sequence type interaction: $\left.F_{(2,42)}=2.69 ; p=0.08\right)$. Additional unpaired $t$ tests confirmed that the $\mathrm{H}$ group had shorter cARTs than the controls on each fixed sequence (all $p$ values $<0.04$ ).

A repeated measures ANOVA indicated that the $\mathrm{H}$ group and controls differed in the number of errors on the fixed sequences (group by sequence type interaction: $F_{(2,42)}=10.45 ; p=0.0002$ ) and in the number of errors on the fixed sequences across sessions (group by sequence type by session interaction: $F_{(18,378)}=2.58$; $p=0.0005$ ) (Fig. $2 D-F)$. As confirmed with unpaired $t$ tests, the $\mathrm{H}$ group was significantly less accurate than the controls on each sequence type (all $p$ values $<0.002$ ).

However, the groups did not differ in their number of omissions on the same sequences (repeated measures of ANOVA for group by sequence type: $F_{(2,42)}=0.57 ; p=0.57$ ), suggesting that both groups were equally motivated to perform the SRT task.

Probe sessions. For each group, repeated measures ANOVAs indicated a significant sequence type versus probe phase interaction on the probes for both cART and error measures (controls: both $F_{(2,24)}$ values $>12.18$, both $p$ values $\leq 0.0002$; H group: both $F_{(2,16)}$ values $>15.85$, both $p$ values $\left.\leq 0.0002\right)$. This suggests that each group differed in their cARTs and errors between the sequence and random-order phase of probe sessions on each sequence type. Next, using paired $t$ tests, the cARTs and the number of errors were compared for each group between the sequence
Reaction Time
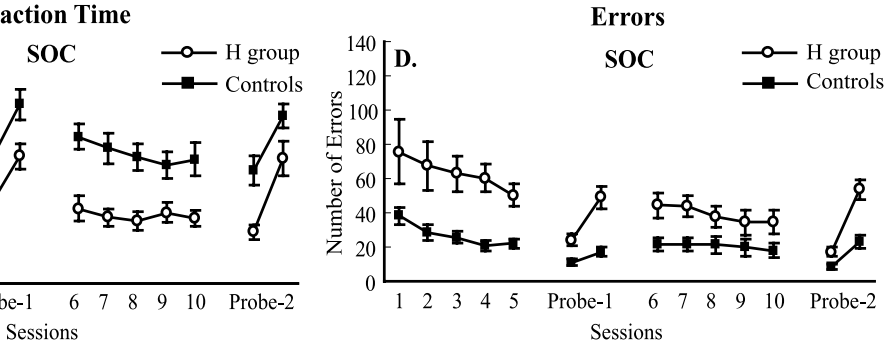

Sessions
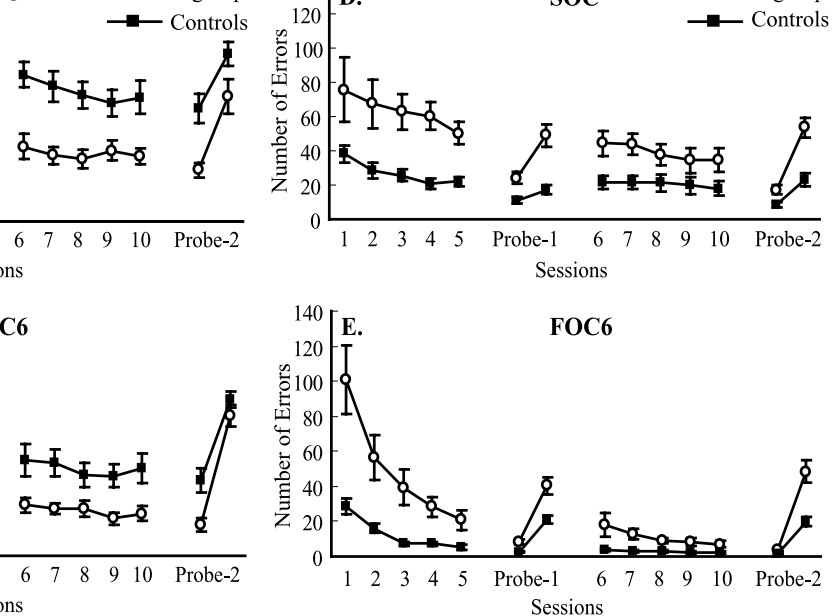

Sessions

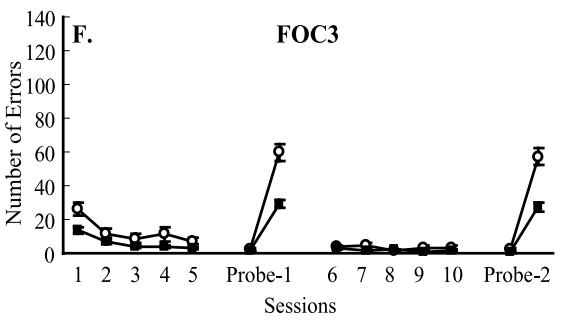

Session

FOC3

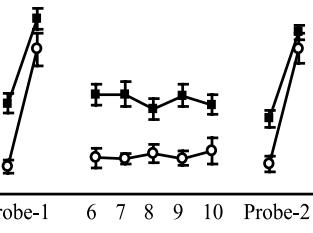

Sessions

on sequence types across testing sessions and probes. $\boldsymbol{A}-\boldsymbol{C}$, Reaction times (CART \pm SEM) of groups on the SOC, FOC6, and FOC3 sequences, respectively. $D-F$, Errors (number of errors \pm SEM) of groups on the $\mathrm{SOC}, \mathrm{FOC6}$, and $\mathrm{FOC} 3$ sequences, respectively.

and the random-order phase on each probe session. On each probe, when the fixed sequence was switched to random-order presentations, both controls and the $\mathrm{H}$ group slowed down significantly (all $p$ values $\leq 0.0009$ ) (Fig. $2 A-C$ ) and made more errors (all $p$ values $<0.02$ ) (Fig. $2 D-F$ ), indicating that both groups showed learning in all three sequences.

A repeated-measures ANOVA confirmed that groups did not differ for omissions across phases of probes (group by sequence type by probe phase: $\left.F_{(2,40)}=1.87 ; p=0.17\right)$, suggesting that groups were equally motivated on the probes. A repeated measures of ANOVA also revealed that group cARTs and errors did not differ between the sequence and random phases across sequence types on the probes (group by sequence type by probe phase: $\operatorname{cARTs}, F_{(2,40)}=2.37, p=0.11$; errors, $F_{(2,40)}=2.95, p=$ $0.06)$, indicating that groups did not differ in the extent or rate of learning on probe sessions.

\section{Comparisons within groups}

Patterns of cARTs and errors on fixed sequences were also compared within each group across the 10 testing sessions. After the initial analysis by repeated-measures ANOVAs on all 10 sessions, testing sessions were grouped into five successive blocks of two sessions for additional comparisons using paired $t$ tests.

Reaction times. A repeated-measures ANOVA indicated that control cARTs differed across sequence types and testing sessions (sequence type by session interaction: $F_{(18,216)}=2.33 ; p=0.002$ ) (Fig. 3A). At each block of testing, controls were equally fast on FOC6 and FOC3 sequences (all $p$ values $>0.2$ ) but were slower on the SOC sequence than on either FOC sequence (all $p$ values $<0.03$ ) except for block 5 , where their speed marginally differed between the SOC and the FOC3 sequences $\left(t_{(12)}=2.16 ; p=\right.$ $0.05)$. This pattern of findings indicates that, despite the differences in sequence length and number of ports used among se- 

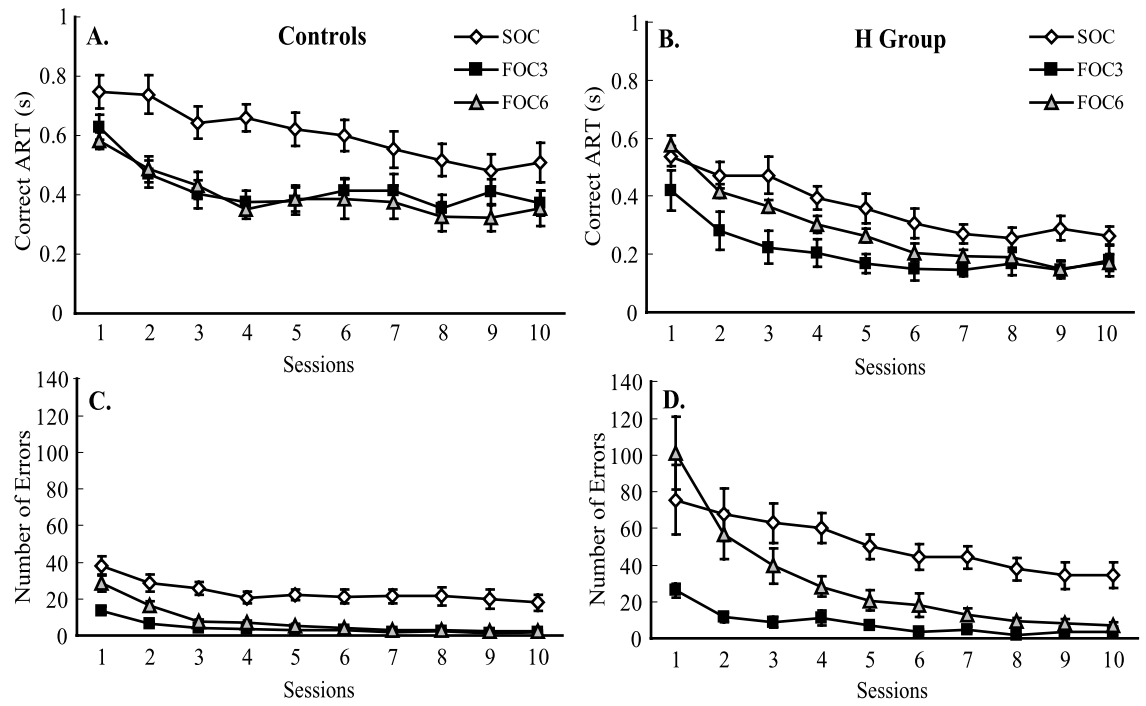

Figure 3. Within-group comparisons of SRT performance on sequence types across testing sessions. $\boldsymbol{A}$, Reaction times (CART \pm SEM) of controls $(n=13)$. $\boldsymbol{B}$, Reaction times of the H group $(n=10)$. C, Errors (number of errors \pm SEM) of controls. $\boldsymbol{D}$, Errors of the $\mathrm{H}$ group.

Table 1. Correct ART (milliseconds \pm SEM) of groups averaged across 10 testing sessions

\begin{tabular}{lllll}
\hline & SOC & FOC6 & FOC3 & Overall \\
\hline Controls $(n=13)$ & $607 \pm 19$ & $400 \pm 16$ & $422 \pm 15$ & $476 \pm 11$ \\
Fast controls $(n=6)$ & $500 \pm 27$ & $290 \pm 19$ & $315 \pm 18$ & $368 \pm 14$ \\
Slow controls $(n=7)$ & $699 \pm 22$ & $495 \pm 19$ & $513 \pm 17$ & $569 \pm 13$ \\
H group $(n=10)$ & $362 \pm 17$ & $283 \pm 15$ & $209 \pm 17$ & $285 \pm 10$ \\
Fast H rats $(n=5)$ & $307 \pm 22$ & $231 \pm 21$ & $122 \pm 16$ & $220 \pm 13$ \\
Slow H rats $(n=5)$ & $417 \pm 23$ & $336 \pm 20$ & $295 \pm 24$ & $350 \pm 13$ \\
\hline
\end{tabular}

quences, controls distinguished second-order and first-order problem solutions but did not differentiate between long and short first-order sequences. That is, controls differentiated sequences by their complexity not by their length.

A repeated-measures ANOVA also indicated that the $\mathrm{H}$ group cARTs differed across sequence types and testing sessions (sequence type by session interaction: $F_{(18,162)}=2.47 ; p=0.001$ ) (Fig. $3 B$ ). However, the $\mathrm{H}$ group differed from the controls in the pattern of performance across the testing blocks. In block $1, \mathrm{H}$ rats had comparable cARTs on the FOC6 and SOC sequences $\left(t_{(9)}=0.23 ; p=0.83\right)$, whereas their cARTs on FOC3 sequence were shorter than on the FOC6 sequence $\left(t_{(9)}=2.96 ; p=0.02\right)$. $\mathrm{H}$ rats also had numerically shorter CARTs on the FOC3 sequence than on the SOC sequence but this difference was not statistically significant $\left(t_{(9)}=1.84 ; p=0.1\right)$. However, at blocks 2 and 3 , the $\mathrm{H}$ group had similar cARTs on the FOC6 and SOC sequences (all $p$ values $>0.12$ ), whereas they had shorter cARTs on the FOC3 sequence than on both of the longer sequence types (all $p$ values $<0.05$ ). This observation suggests that the $\mathrm{H}$ group initially distinguished sequences based on their lengths alone. In block $4, \mathrm{H}$ rats had comparable cARTs on SOC and FOC6 sequences $\left(t_{(9)}=\right.$ 2.07; $p=0.07)$ and their cARTs were shorter on the FOC3 sequence than on the SOC sequence $\left(t_{(9)}=4.61, p=0.001\right)$ but their speed did not differ on the two FOC sequences $\left(t_{(9)}=1.79 ; p=\right.$ 0.11 ). Finally, in block 5 , the $H$ group had equivalent cARTs on the two FOC sequences $\left(t_{(9)}=0.09 ; p=0.93\right)$ but were slower on the SOC sequence than either FOC sequence (all $p$ values $<0.02$ ). These findings suggest that the $\mathrm{H}$ group eventually distinguished the se- quences based on their complexity alone, similar to the controls albeit after more extensive training.

The overall pattern of findings suggests that the controls and the $\mathrm{H}$ group may have used different strategies to solve the different types of sequence problems. Whereas controls distinguished the sequences based on their complexity alone throughout training, $\mathrm{H}$ rats initially distinguished sequences only by their length, and then gradually acquired the ability to distinguish the sequences based on their complexity rather than length. Because the H group had shorter cARTs than controls on all sequences, one possibility is that the different cART patterns could have resulted from group differences in overall speed and not from differences in strategy. To test this possibility, we separated each group of rats into "fast" and "slow" using a median split: for each rat, session cARTs were averaged across three sequences (overall cART) (Table 1). In each group, half of the rats with the shorter overall cARTs were designated as fast rats whereas the other half with the longer overall cARTs were designated as slow rats. Subsequently, we compared cART patterns for the three types of sequences in each group.

In each group, fast and slow rats displayed cART patterns similar to their entire group. Furthermore, when subsets of the control and $\mathrm{H}$ rats were matched for speed, the group cART patterns were still different. The overall cART scores of fast controls $(n=6)$ and slow $\mathrm{H}$ rats $(n=5)$ on fixed sequences were $368 \pm 15 \mathrm{~ms}$ (mean \pm SEM) and $350 \pm 13 \mathrm{~ms}$, respectively (Table $1)$. An unpaired $t$ test confirmed that groups did not differ in overall cART $\left(t_{(9)}=0.93 ; p=0.38\right)$. Nevertheless, fast controls distinguished the sequences based on their complexity in early blocks of training, whereas slow $\mathrm{H}$ rats approached all sequence problems similarly, albeit numerically resembling the entire $\mathrm{H}$ group. This indicates that performance speed alone cannot explain the cART differences between groups.

Errors. In controls, a repeated measures ANOVA on errors did not indicate an interaction between sequence type versus sessions $\left(F_{(18,216)}=1.13 ; p=0.33\right)$ but indicated a significant sequence type effect $\left(F_{(2,24)}=55.55 ; p<0.0001\right)$ and a significant session effect $\left(F_{(9,108)}=24.04 ; p<0.0001\right)$ (Fig. 3C). Additional analyses by paired $t$ tests indicated that in block 1, controls made similar errors on longer sequences $\left(t_{(12)}=2.11 ; p=0.06\right)$ and they made less errors on the FOC3 sequence than on either long sequence (all $p$ values $<0.003$ ). In block 2 , controls made more errors on the SOC sequence than on both of the FOC sequences (all $p$ values $\leq 0.0001$ ) and they also made more errors on the FOC6 than on the FOC3 sequence $\left(t_{(12)}=3.76 ; p=0.003\right)$. From blocks 3-5, controls continued to make more errors on SOC than on either FOC sequence (all $p$ values $<0.005$ ) but their errors did not differ between FOC sequences (all $p$ values $>0.12$ ). These findings indicate that control error patterns initially distinguished the length of the sequences in block 1 and quickly differentiated only the complexity of the sequences starting in block 3 and thereafter.

A repeated-measures ANOVA indicated that the error rates of the $\mathrm{H}$ group differed between sequence types and testing sessions (sequence type by session interaction: $F_{(18,162)}=3.46 ; p<$ 0.0001 ) (Fig. $3 D$ ), indicating that the pattern of errors differed 
across the testing blocks in the $\mathrm{H}$ group. In block $1, \mathrm{H}$ rats made a similar number of errors on the SOC and the FOC6 sequences $\left(t_{(9)}=0.32 ; p=0.75\right)$, whereas they made less errors on FOC3 than on both of the longer sequences (all $p$ values $\leq 0.006$ ). From block $2-4$, the $\mathrm{H}$ group made more errors on the SOC sequence than on either FOC sequence (all $p$ values $<0.007$ ) and their number of errors on FOC6 were greater than that on FOC3 (all $p$ values $<0.03$ ). Finally, in block 5 , the $\mathrm{H}$ group made more errors on the SOC sequence than on either FOC sequence (all $p$ values $<0.002)$ and their number of errors did not differ on either of the FOC sequences $\left(t_{(9)}=1.84 ; p=0.1\right)$. These findings indicate that the $\mathrm{H}$ group error patterns initially differentiated sequences only by their length in block 1 . From block 2 to 4 , the H group distinguished sequence complexity in addition to sequence length. Finally, the $\mathrm{H}$ group error pattern discriminated sequence complexity alone in the last block of testing.

Similar to the cART patterns, the different group patterns on errors also suggest that the controls and the $\mathrm{H}$ group may have used different strategies to solve the different types of sequence problems. At first, error patterns in the controls distinguished the sequences based on their length and started distinguishing complexity in addition to sequence length in the second block. Starting in block 3 and consistently thereafter, the accuracy of the control group quickly depended on only the complexity of the sequence problems. However, the error patterns of $\mathrm{H}$ rats initially distinguished sequences only by their length, and then gradually distinguished the sequences also based on their complexity in addition to their length. However, the $\mathrm{H}$ group eventually distinguished the sequences based on their complexity alone in block 5 , similar to the controls, albeit after more extensive training.

In each group, both fast and slow rats displayed similar error patterns as their entire group. Moreover, when subsets of the controls and $\mathrm{H}$ rats were matched for speed, the group error patterns were still different. Fast controls initially distinguished sequences by length but rapidly discriminated the sequences only by their complexity in block 3 and consistently thereafter. However, although slow $\mathrm{H}$ rats also differentiated the sequences by their length at first, they did not distinguish sequences consistently depending on their complexity alone across subsequent blocks of testing. This also indicates that, similar to the group cART pattern comparisons, performance speed alone cannot elucidate the group error differences on different types of sequences.

\section{A test-order effect}

In the present study, the order of the sequences was partially counterbalanced across separate groups of rats to overcome possible test-order effects on performance. We examined the effect of test order in the controls by comparing the data for each sequence among three testing-groups.

A repeated-measures of ANOVA indicated that the three testing groups of controls differed in cARTs across sequences (test group by sequence type interaction: $\left.F_{(4,20)}=4.93 ; p=0.006\right)$. However, a repeated measures of ANOVA indicated that the three groups of controls did not differ in cARTs across phases of probes across sequences (test group by sequence type by probe phase interaction: $\left.F_{(4,20)}=2.25 ; p=0.1\right)$.

A repeated-measures of ANOVA on errors indicated that the three testing-groups of controls did not differ in number of errors across sequences (test group by sequence type interaction: $F_{(4,20)}=$ $1.14 ; p=0.37)$. However, a repeated measures of ANOVA revealed significant group differences in errors across phases of probes across sequences (test group by sequence type by probe phase interaction: $\left.F_{(4,20)}=4.0 ; p=0.02\right)$.
These findings indicate that the three groups of controls displayed some differences in reaction time on testing sessions and in the number of errors on probes when the fixed sequence was switched to random presentations. Notably, any testing order effect on the control and $\mathrm{H}$ group differences was minimized by our counterbalanced experimental design.

\section{Discussion}

The present findings indicate that the hippocampal formation itself plays an essential role in the rapid learning of higher-order sequential associations. We found that normal rats, like normal humans, fully acquire higher-order sequence structures within two training sessions, whereas rats with hippocampal formation damage, like amnesic patients with more widespread MTL damage, are impaired in higher-order sequence learning and initially treat simple and complex sequences equivalently (Curran, 1997). Both controls and rats with hippocampal lesions learned firstand second-order sequences as indicated by a decrease in reaction time and in the number of errors across multiple testing sessions, as well as by an increase in reaction times when fixed sequences were switched to random-order on probes. Also, both groups eventually distinguished between first-order and second-order sequences in their reaction time and error patterns. However, the control and the $\mathrm{H}$ groups differed in the amount of training required to solve the sequence problems and in the number of errors in training, suggesting differences in the underlying basis of sequence learning. As a general trend, controls were slower and more accurate, whereas the $\mathrm{H}$ group was faster and less accurate in their responses on all sequences. Furthermore, during training, controls quickly distinguished the sequences based on their complexity alone, whereas the $\mathrm{H}$ group initially differentiated the sequences based on their length and only gradually distinguished the sequences by their complexity. These observations indicate that extrahippocampal structures can support both first-order and higher-order sequence learning, but acquisition of more complex associations required for second-order sequence learning is gradual within extrahippocampal systems.

\section{Speed and accuracy}

To the extent that sequence learning is reflected in the difference between RTs on sequence and random order blocks, the two groups learned the sequences equally well. However, this apparent normal learning occurred at a cost: $\mathrm{H}$ rats made more errors than controls on each type of sequence. Therefore, sequence learning by $\mathrm{H}$ rats can be characterized as reflecting improved performance of a system that supports the acquisition of stereotyped sequential behavioral patterns in the absence of the influence of another system that supports greater accuracy.

$\mathrm{H}$ rats had consistently shorter RTs than controls during learning of each type of sequence. Does the increase in response speed in sequential behavior account for the selective effects of hippocampal damage on higher-order SRT learning? Our comparisons of learning rates by $\mathrm{H}$ and control rats across different types of sequences indicate that this was not the case. Both fast and slow control rats judged the sequences based on their complexity in early blocks of testing, whereas both fast and slow $\mathrm{H}$ rats initially distinguished sequences based on length. Similarly, previous SRT studies with human amnesics have not revealed consistent results on response speed and its relationship to accuracy. Some studies reported that Korsakoff's patients were significantly slower than the control subjects on the SRT task, and in only one of these studies was FOC learning less accurate (Nissen and Bullemer, 1987; Nissen et al., 1989). Another study reported faster 
response speeds during the initial blocks of testing among a mixed group of Alzheimer's patients and patients with other etiologies including anoxia, encephalitis, and stroke and only a marginal difference in accuracy (Curran, 1997). Reber and Squire (1998) did not observe statistically significant differences in response speed between controls and amnesic patients with Korsakoff s disease or bilateral hippocampal damage. The variability observed in response speed and accuracy among the studies on amnesic patients may have been a result of the different brain pathologies among their amnesic subjects. In the present study, the lesions were similar among the subjects and all were restricted to the hippocampal formation. Still, we found that some subjects have faster response speeds than controls, whereas others did not. Despite these differences in response speed, the pattern on effects of hippocampal damage was similar among different types of sequences, so alterations in response speed cannot account for the selective effects of hippocampal damage on higherorder sequence learning.

\section{The role of the hippocampal formation in motor-sequence learning}

Curran (1997) investigated the acquisition of first- and second-order sequences in humans using an SRT design that involved presentations of sequence and random trials mixed within each block. Curran (1997) found that both amnesic patients and controls acquired the first- and second-order sequences, as reflected in increased reaction times for random-order versus repeated-sequence presentations. However, when the reaction time for the same pair-wise transition was compared between the sequence and random trials, amnesic patients were impaired only on the second-order transitions compared with control subjects. Furthermore, whereas normal subjects had a greater differential in reaction times for SOC than FOC sequences, the amnesic patients had similar increases in reaction time on both types of sequences. The present findings are consistent with Curran's observations on human amnesic patients. Similar to Curran's observations, here we found that both control subjects and the $\mathrm{H}$ group learned the FOC and SOC sequences, as reflected in increased response latencies on probe trials. However, whereas the controls' pattern of learning distinguished first- and second-order sequences, the $\mathrm{H}$ group pattern initially distinguished sequences by their length and not by their complexity.

Consistent with the findings on amnesic patients, Schendan et al. (2003) found that the MTL structures, including the hippocampus and the surrounding cortices, were activated when normal subjects learned an SOC sequence, regardless of awareness of the sequence. Moreover, the MTL activation was stronger earlier during learning. This finding is entirely consistent with the present observation of an important role for the hippocampal formation in acquiring higher-order associations early in sequence training.

A key issue concerns what structures are essential to rapid learning of SOC sequences. Previous reports on SRT learning with SOC sequences have focused on amnesic patients with various etiologies, including Korsakoff's disease, herpes simplex encephalitis, anoxia, and thalamic infarct, wherein damage is not limited to the hippocampal formation (Curran, 1997; Reber and Squire, 1998; Hopkins et al., 2004). Also, functional imaging during SRT performance that shows activation of the hippocampal formation has also revealed activation of the parahippocampal cortex as well as other cortical areas (Willingham et al., 2002; Schendan et al., 2003). These and other findings led Keele et al. (2003) to propose the existence of a widespread network of cortical areas that mediate higher-order sequence learning. The present results provide new evidence that the hippocampal formation itself is an essential element of the network that supports the rapid learning of higher-order sequences. Furthermore, the findings of DeCoteau and Kesner (2000) indicating that rats with hippocampal lesions are selectively impaired in "explicit" expression of a sensorimotor sequence, combined with the present finding that rats with damage to the hippocampal formation are also selectively impaired in higher-order sequence learning, suggests a linkage between the capacities for explicit memory and for rapid learning of complex sequences of information (Eichenbaum, 2004).

\section{References}

Agster KL, Fortin NJ, Eichenbaum H (2002) The hippocampus and disambiguation of overlapping sequences. J Neurosci 22:5760-5768.

Burgess N, Maguire EA, O'Keefe J (2002) The human hippocampus and spatial and episodic memory. Neuron 35:625-641.

Curran T (1997) Higher-order associative learning in amnesia: evidence from the serial reaction time task. J Cogn Neurosci 9:522-533.

DeCoteau WE, Kesner RP (2000) A double dissociation between the rat hippocampus and medial caudoputamen in processing two forms of knowledge. Behav Neurosci 114:1096-1108.

Eichenbaum H (2004) Hippocampus: cognitive processes and neural representations that underlie declarative memory. Neuron 44:109-120.

Ergorul C, Eichenbaum H (2004) The hippocampus and memory for "what," "where," and "when." Learn Mem 11:397-405.

Fortin NJ, Agster KL, Eichenbaum HB (2002) Critical role of the hippocampus in memory for sequences of events. Nat Neurosci 5:458-462.

Hopkins RO, Waldram K, Kesner RP (2004) Sequences assessed by declarative and procedural tests of memory in amnesic patients with hippocampal damage. Neuropsychologia 42:1877-1886.

Jackson GM, Jackson SR, Harrison J, Henderson L, Kennard C (1995) Serial reaction time learning and Parkinson's disease: evidence for a procedural learning deficit. Neuropsychologia 33:577-593.

Keele SW, Mayr U, Ivry R, Hazeltine E, Heuer H (2003) The cognitive and neural architecture of sequence representation. Psychol Rev 110:316-339.

Kesner RP, Gilbert PE, Barua LA (2002) The role of the hippocampus in memory for the temporal order of a sequence of odors. Behav Neurosci 116:286-290.

Knopman DS, Nissen MJ (1987) Implicit learning in patients with probable Alzheimer's disease. Neurology 37:784-788.

Knopman DS, Nissen MJ (1991) Procedural learning is impaired in Huntington's disease: evidence from the serial reaction time task. Neuropsychologia 29:245-254.

Lee AK, Wilson MA (2002) Memory of sequential experience in the hippocampus during slow wave sleep. Neuron 36:1183-1194.

Levy WB (1989) A computational approach to hippocampal function. In: Computational models of learning in simple systems. (Hawkins RD, Bower GH, eds), pp 243-305. New York: Academic.

Lisman JE (1999) Relating hippocampal circuitry to function: Recall of memory sequences by reciprocal dentate-CA3 interactions. Neuron 22:233-242.

Manns JR, Squire LR (2001) Perceptual learning, awareness, and the hippocampus. Hippocampus 11:776-782.

Nissen MJ, Bullemer P (1987) Attentional requirements of learning: Evidence from performance measures. Cognit Psychol 19:1-32.

Nissen MJ, Willingham D, Hartman M (1989) Explicit and implicit remembering: when is learning preserved in amnesia? Neuropsychologia 27:341-352.

Paxinos G, Watson C (1997) The rat brain in stereotaxic coordinates. San Diego: Academic.

Reber PJ, Squire LR (1994) Parallel brain systems for learning with and without awareness. Learn Mem 1:217-229.

Reber PJ, Squire LR (1998) Encapsulation of implicit and explicit memory in sequence learning. J Cogn Neurosci 10:248-263.

Schendan HE, Searl MM, Melrose RJ, Stern CE (2003) An fMRI study of the role of the medial temporal lobe in implicit and explicit sequence learning. Neuron 37:1013-1025.

Tulving E (1972) Episodic and semantic memory. In: Organization of memory (Tulving E, Donaldson W, eds), pp 381-403. New York: Academic.

Wallenstein GV, Eichenbaum H, Hasselmo ME (1998) The hippocampus as an associator of discontiguous events. Trends Neurosci 21:317-323.

Willingham DB, Salidis J, Gabrieli JD (2002) Direct comparison of neural systems mediating conscious and unconscious skill learning. J Neurophysiol 88:1451-1460. 